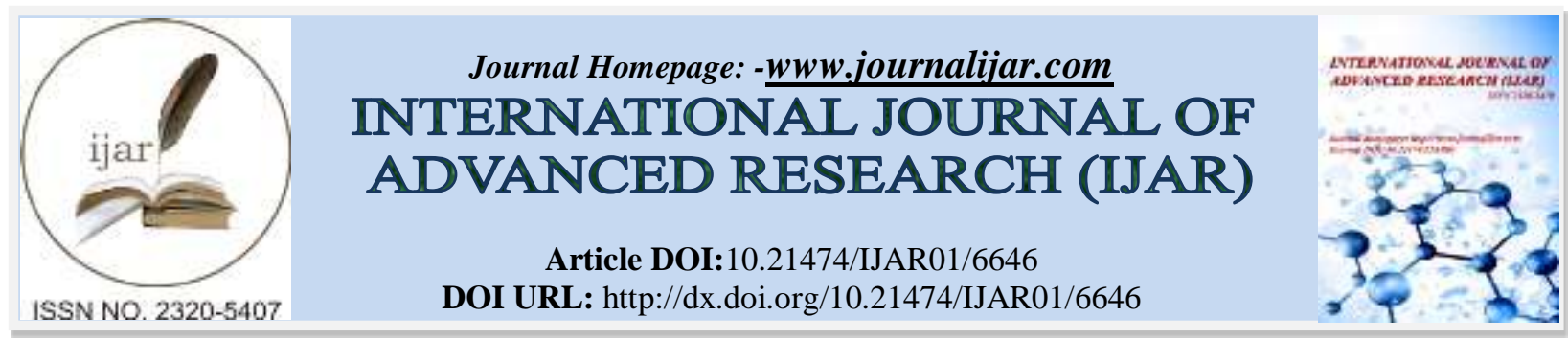

RESEARCH ARTICLE

\title{
OPTICAL PROPERTIES OF QUANTUM WIRE SUPERLATTICES FOR AN INCIDENCE PLANE PARALLEL TO THE LAYERS.
}

\author{
Raihane Charguia $^{1,2}$, Hela Fenniche ${ }^{1}$ and Raouf Bennaceur ${ }^{1}$. \\ 1. Department of Physics, Faculty of Science and Arts at Buraidah, Qassim University, Saudi Arabia. \\ 2. Laboratory of Condensed Matter Physics, Department of Physics, Faculty of Science, University of Tunis El \\ Manar II 2092 Tunis, Tunisia.
}

\section{Manuscript Info}

Manuscript History

Received: 02 January 2018

Final Accepted: 04 February 2018

Published: March 2018

\section{Abstract}

The optical reflectivity and transmission in semiconductor quantum wire grating, are considered theoretically for an incidence parallel to the layers. The curves are obviousness in favour of coupled TE and TM band gap energy with very high reflectivity for $\theta>80^{\circ}$ and which is accompanied by a nearly vanishing transmittance. In such an extended energy range, the system behaves as a mirror.

Copy Right, IJAR, 2018,. All rights reserved.

\section{Introduction:-}

The semiconductors, having both a high refractive index and a low absorption, combined with air are an ideal choice for the production of photonic band gap materials. It should be noted that the width of a photonic band gap of a material is even wider than the refractive index contrast between the materials of which it is composed is high. Indeed, the strong contract of index allows a good vertical confinement of the light, and then the out-of-plane losses are limited.

The electronic and optical properties of inhomogeneous structure such as grating of quantum wires are intensively studied experimentally and theoretically [1-8]. In semiconductor insulator cylindrical quantum wires, both experimental and theoretical investigations of optical properties are presented by E. A. Muljarov et al [1]. These experimental and calculated absorption and exciton transition energies, which demonstrate the prominent excitonic behavior, are in good agreement. The $s$ - and $p$-polarized spectra reflectivity of grating of rectangular quantum well wires is computed by using Green's function formalism [2]. For an incidence plane perpendicular to the layers, the calculations of the reflectivity spectra vs the energy of the incident electromagnetic wave for $s$ - and $p$-polarized light are signs of the opening of a photonic band gap over a whole range of energy [3].

Also, for a superlattice of quantum wires, spectra polariton dispersions and their radiative decay rates were discussed [4]. All these results were fundamental for the study of the optical properties of these structure for an incidence plane parallel to the layers of the grating $(\varphi=\pi / 2)$.

In this paper we focus on the calculations of the reflectance and transmittance in semiconductor quantum wires grating when the plan of incidence is parallel to the wires. In section 2 we present our model. The resulting reflectivity spectra are discussed in section 3. Section 4 is devoted to the conclusion.

\section{Theoretical framework:-}

In this section we focus on the propagation of an electromagnetic wave inside an inhomogeneous medium such as dielectric quantum wire grating. The proposed theoretical models show that the light propagation is widely 
influenced by the structure of interfaces. In our work, and in order to simplify the physical problem, we neglect imperfections and defects at the interfaces and we assume that our system is one-dimensional.

For $0<z<L_{z}$, the unperturbed electric field resulting from guided modes generated by the dielectric grating having the following expression:

$$
E_{0 i}(x, y, z)=\sum_{n}\left(A_{i n} e^{i k_{n} z}+B_{i n} e^{-i k_{n} z}\right) \sum_{G} E_{0 i}\left(k_{x}+G, k_{y}, k_{n}\right) e^{i\left(k_{x}+G\right) x} e^{i k_{y} y}
$$

where $i=x, y, z$

For parallel incidence to the layers, the total electric field in the vacuum $(z<0)$ actually includes two terms: the first one is relating to the incident plane wave and the second is attributed to the reflected waves, namely:

$$
\begin{aligned}
& E_{x(y)}(x, y, z)=\sum_{G}\left(\delta_{G, 0} e^{i q_{z}(G) z}+r_{G}^{s(p)} e^{-i q_{z}(G) z}\right) e^{i\left(k_{x}+G\right) x} e^{i k_{y} y} \\
& E_{z}(x, y, z)=\sum_{G}\left(-\frac{\left(k_{x}+G\right)+k_{y}}{q_{z}(G)} \delta_{G, 0} e^{i q_{z}(G) z}+\frac{\left(k_{x}+G\right) r_{G}^{s}+k_{y} r_{G}^{p}}{q_{z}(G)} e^{-i q_{z}(G) z}\right) e^{i\left(k_{x}+G\right) x} e^{i k_{y} y} \\
& \text { with } q_{z}(G)=\left[\frac{\omega^{2}}{c^{2}} \varepsilon_{0}-\left(k_{x}+G\right)^{2}-k_{y}^{2}\right]^{1 / 2}
\end{aligned}
$$

In the substrate $(z>h)$, the transmitted electric field is:

$$
\begin{aligned}
& E_{x(y)}(x, y, z)=\sum_{G} t_{G}^{s(p)} e^{i k_{z}(G) z} e^{i\left(k_{x}+G\right) x} e^{i k_{y} y} \\
& E_{z}(x, y, z)=\sum_{G}-\frac{\left(k_{x}+G\right) t_{G}^{s}+k_{y} t_{G}^{p}}{k_{z}(G)} e^{i k_{z}(G) z} e^{i\left(k_{x}+G\right) x} e^{i k_{y} y} \\
& \text { with } k_{z}(G)=\left[\frac{\omega^{2}}{c^{2}} \varepsilon_{b}-\left(k_{x}+G\right)^{2}-k_{y}^{2}\right]^{1 / 2}
\end{aligned}
$$

The coefficients $r_{0 G}^{s}, r_{0 G}^{p}, t_{0 G}^{s}$ and $t_{0 G}^{p}$ are determined from the boundary conditions imposed on the electric field components at $z=0$ and $z=h$, namely:

at $z=0$

$$
\begin{aligned}
& r_{0 G}^{s}=\sum_{n}\left(A_{x n}+B_{x n}\right) E_{x}\left(G, k_{y}, k_{n}\right)-\delta_{G, 0} \\
& r_{0 G}^{p}=\sum_{n}\left(A_{y n}+B_{y n}\right) E_{0 y}\left(G, k_{y}, k_{n}\right)-\delta_{G, 0}
\end{aligned}
$$

at $z=h$

$$
\begin{aligned}
& t_{0 G}^{s} e^{i k_{z}(G) h}=\sum_{n}\left(A_{x n} e^{i k_{n} h}+B_{x n} e^{-i k_{n} h}\right) E_{0 x}\left(k_{x}+G, k_{y}, k_{n}\right) \\
& t_{0 G}^{p} e^{i k_{z}(G) h}=\sum_{n}\left(A_{y n} e^{i k_{n} h}+B_{y n} e^{-i k_{n} h}\right) E_{0 y}\left(k_{x}+G, k_{y}, k_{n}\right)
\end{aligned}
$$

where $A_{x n}=A_{y n} \quad, \quad B_{x n}=B_{y n}$ 
The coefficients $A_{x n}$ and $B_{x n}$ are determined from the boundary conditions imposed on the components of the magnetic field $\vec{B}$. In our calculations, we will neglect only all surface currents. The continuity of the tangential component of the magnetic field in $z=0$ and $z=h$ leads to:

For $s$ - polarized light,

at $z=0$

$$
\begin{aligned}
& 2\left(q_{z}(G)+\frac{\left(k_{x}+G\right)^{2}}{q_{z}(G)}+\frac{k_{y}\left(k_{x}+G\right)}{q_{z}(G)}\right) \delta_{G, 0}= \\
& \sum_{n}\left[\left(q_{z}(G)+\frac{\left(k_{x}+G\right)^{2}}{q_{z}(G)}+k_{n}\right) E_{0 x}\left(G, q_{y}, k_{n}\right)+\frac{k_{y}\left(k_{x}+G\right)}{q_{z}(G)} E_{0 y}\left(G, k_{y}, k_{n}\right)-\left(k_{x}+G\right) E_{0 z}\left(G, k_{y}, k_{n}\right)\right] A_{x n} \\
& +\sum_{n}\left[\left(q_{z}(G)+\frac{\left(k_{x}+G\right)^{2}}{q_{z}(G)}-k_{n}\right) E_{0 x}\left(G, q_{y}, k_{n}\right)+\frac{k_{y}\left(k_{x}+G\right)}{q_{z}(G)} E_{0 y}\left(G, k_{y}, k_{n}\right)+\left(k_{x}+G\right) E_{0 z}\left(G, k_{y}, k_{n}\right)\right] B_{x n}
\end{aligned}
$$

at $z=h$

$\sum_{n}\left[\left(k_{z}(G)+\frac{\left(k_{x}+G\right)^{2}}{k_{z}(G)}-k_{n}\right) E_{0 x}\left(G, q_{y}, k_{n}\right)+\frac{k_{y}\left(k_{x}+G\right)}{q_{z}(G)} E_{0 y}\left(G, k_{y}, k_{n}\right)+\left(k_{x}+G\right) E_{0 z}\left(G, k_{y}, k_{n}\right)\right] A_{x n} e^{i k_{n} h}+$

$\sum_{n}\left[\left(k_{z}(G)+\frac{\left(k_{x}+G\right)^{2}}{k_{z}(G)}+k_{n}\right) E_{0 x}\left(G, q_{y}, k_{n}\right)+\frac{k_{y}\left(k_{x}+G\right)}{k_{z}(G)} E_{0 y}\left(G, k_{y}, k_{n}\right)-\left(k_{x}+G\right) E_{0 z}\left(G, k_{y}, k_{n}\right)\right] B_{x n} e^{-i k_{n} h}$

$=0$

For $p$ - polarized light,

at $z=0$

$$
\begin{aligned}
& 2\left(q_{z}(G)+\frac{k_{y}\left(k_{x}+G\right)}{q_{z}(G)}+\frac{k_{y}^{2}}{q_{z}(G)}\right) \delta_{G, 0}= \\
& \sum_{n}\left[\frac{k_{y}\left(k_{x}+G\right)}{q_{z}(G)} E_{0 x}\left(G, k_{y}, k_{n}\right)+\left(q_{z}(G)+\frac{k_{y}^{2}}{q_{z}(G)}+k_{n}\right) E_{0 y}\left(G, k_{y}, k_{n}\right)-k_{n} E_{0 z}\left(G, k_{y}, k_{n}\right)\right] A_{x n}+ \\
& \sum_{n}\left[\frac{k_{y}\left(k_{x}+G\right)}{q_{z}(G)} E_{0 x}\left(G, k_{y}, k_{n}\right)+\left(q_{z}(G)+\frac{k_{y}^{2}}{q_{z}(G)}-k_{n}\right) E_{0 y}\left(G, k_{y}, k_{n}\right)+k_{n} E_{0 z}\left(G, k_{y}, k_{n}\right)\right] B_{x n} \\
& \text { at } \quad z=h \\
& \sum_{n}\left[\frac{k_{y}\left(k_{x}+G\right)}{k_{z}(G)} E_{0 x}\left(G, k_{y}, k_{n}\right)+\left(k_{z}(G)+\frac{\left(k_{x}+G\right)^{2}}{k_{z}(G)}-k_{n}\right) E_{0 y}\left(G, k_{y}, k_{n}\right)+k_{y} E_{0 z}\left(G, k_{y}, k_{n}\right)\right] A_{x n} e^{i k_{n} h}+ \\
& \sum_{n}\left[\frac{k_{y}\left(k_{x}+G\right)}{k_{z}(G)} E_{0 x}\left(G, k_{y}, k_{n}\right)+\left(k_{z}(G)+\frac{\left(k_{x}+G\right)^{2}}{k_{z}(G)}+k_{n}\right) E_{0 y}\left(G, k_{y}, k_{n}\right)-k_{y} E_{0 z}\left(G, k_{y}, k_{n}\right)\right] B_{x n} e^{-i k_{n} h} \\
& =0
\end{aligned}
$$

The solving of these linear equations yields to the evaluation of reflection coefficient $\left(r_{0 G}^{s}, r_{0 G}^{p}\right)$ and transmission coefficients $\left(t_{0 G}^{s}, t_{0 G}^{p}\right)$ for an incidence parallel to the layers where the corresponding incidence plane is $(y, z)$. In this 
case, the $T E$ and $T M$ polarisations are coupled. The reflectivity $\mathrm{R}$ and the transmittance $T$, derived from the averaged of Poynting vector, are given by:

$$
\begin{aligned}
& R=\sum_{G} \frac{q_{z}(0)}{q_{z}(G)} \frac{\left(q_{z}^{2}(G)+\left(k_{x}+G\right)^{2}\right)\left|r_{0 G}^{s}\right|^{2}+\left(q_{z}^{2}(G)+k_{y}^{2}\right)\left|r_{0 G}^{p}\right|^{2}+k_{y}\left(k_{x}+G\right)\left(r_{0 G}^{s} \bar{r}_{0 G}^{p}+r_{0 G}^{p} \bar{r}_{0 G}^{s}\right)}{2 q_{z}^{2}(0)+\left(q_{x}+q_{y}\right)} \\
& T=\sum_{G} \frac{q_{z}(0)}{k_{z}(G)} \frac{\left(k_{z}^{2}(G)+\left(k_{x}+G\right)^{2}\right)\left|t_{0 G}^{s}\right|^{2}+\left(k_{z}^{2}(G)+k_{y}^{2}\right)\left|t_{0 G}^{p}\right|^{2}+k_{y}\left(k_{x}+G\right)\left(t_{0 G}^{s} \bar{t}_{0 G}^{p}+t_{0 G}^{p} \bar{t}_{0 G}^{s}\right)}{2 q_{z}^{2}(0)+\left(k_{x}+k_{y}\right)}
\end{aligned}
$$

\section{Numerical results:-}

Let us consider a grating of GaAs quantum wires, where the $y$ direction is parallel to the wire axis. We denote by $L_{x}$ the lateral dimension of the rectangular wire, $d$ the periodicity of the grating and $h$ is its vertical thickness. $\varepsilon_{0}$ is the dielectric constant of the vacuum space and $\varepsilon_{b}$ refractive index of the wires. As described later, the high index contrast is a major interest in the development of energy bands prohibiting the propagation of light.

The system is illuminated by an electromagnetic wave propagating in oblique incidence. The polar and azimuthal angles of incidence $\theta$ and $\varphi$ specify the incoming light incidence. In this case, the situation becomes more complex. Indeed, for oblique incidence propagation the apparent optical thickness of the layers exchanges. The plane of incidence is considered parallel to the layers, namely, $\varphi=\pi / 2$. The wavelength is chosen to be $\lambda=790 \mathrm{~nm}$. It is interesting to note that in our calculations of the reflectivity rate, our starting point is to optimize parameters of our structure in order to obtain a good reflection (Fig.1). First, we fixed the lateral width of the GaAs layer at $L_{x}=\lambda / 4$ and were optimized width of the period. As shown in (Fig.1), a good reflectivity, over a wide range of energies, is observed for $d$ of the order of the wavelength $(d=\lambda)$.

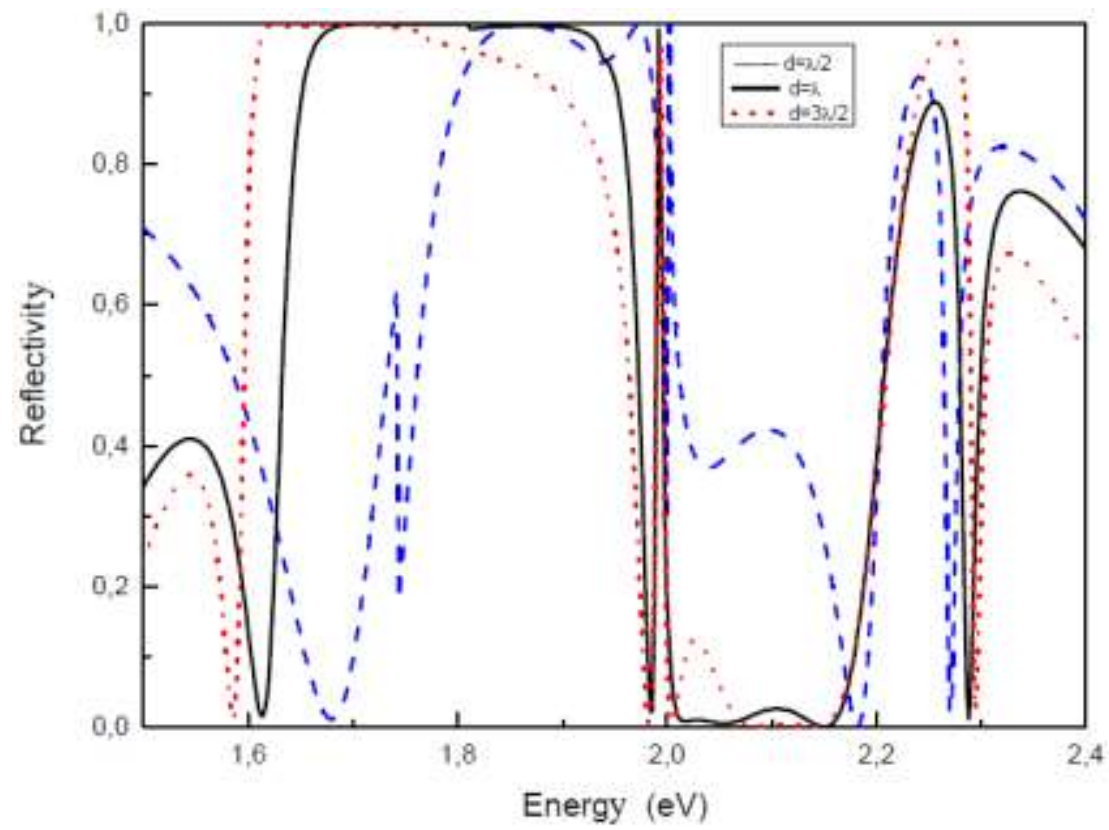

Fig.1:- Reflectivity spectra of semiconductor GaAs quantum grating for $L_{x}=\lambda / 4, h=350 \mathrm{~nm}, \theta=83^{\circ}$ and $\varphi=\pi / 2$.

The curves of figure (Fig.2) show the light reflection rate function of incident energy for different values of the angle of incidence $\theta$. The reflectivity spectra reveal a band of high reflectivity $(R=1)$ from $\left(\theta=80^{\circ}\right)$ which is 
accompanied by a low transmission. This behavior is an evidence for the presence of a photonic band gap. The region of the full band gap matches the intersection of TE and TM band gaps and is extended on the energy band $[1.6 \mathrm{eV}, 2 \mathrm{eV}]$. By increasing the angle of incidence $\theta$, we find that the energies of the edges of the band gap shift slightly towards higher energy and we notice a gradual increase in the reflectivity rate until saturation unit. As the width of the forbidden band, it undergoes a slight enlargement and seems to depend on the angle $\theta$. But when the incident angle decreases beyond a certain angle $\left(\theta<75^{\circ}\right)$, the TE and TM band gaps are no longer overlap and the propagation of the wave is again authorized (low reflectivity rate), while at normal incidence, one finds the Bragg reflections.

As shown in (Fig.2), the reflectivity spectra reveal an important behavior: the opening of a mini-band transmission that appears locate in the band gap. In fact, different types of waves, reflected and transmitted, propagate inside the grating [4] and can interfere constructively for the same phase waves. For this reason, the transmission approaches one. This result is suggestive of the existence of allowed modes in the photonic band gap and shows great similarity with experimental and theoretical observations in photonic crystal defects. This opens the way to realize new components, such as lasers and optical filters.

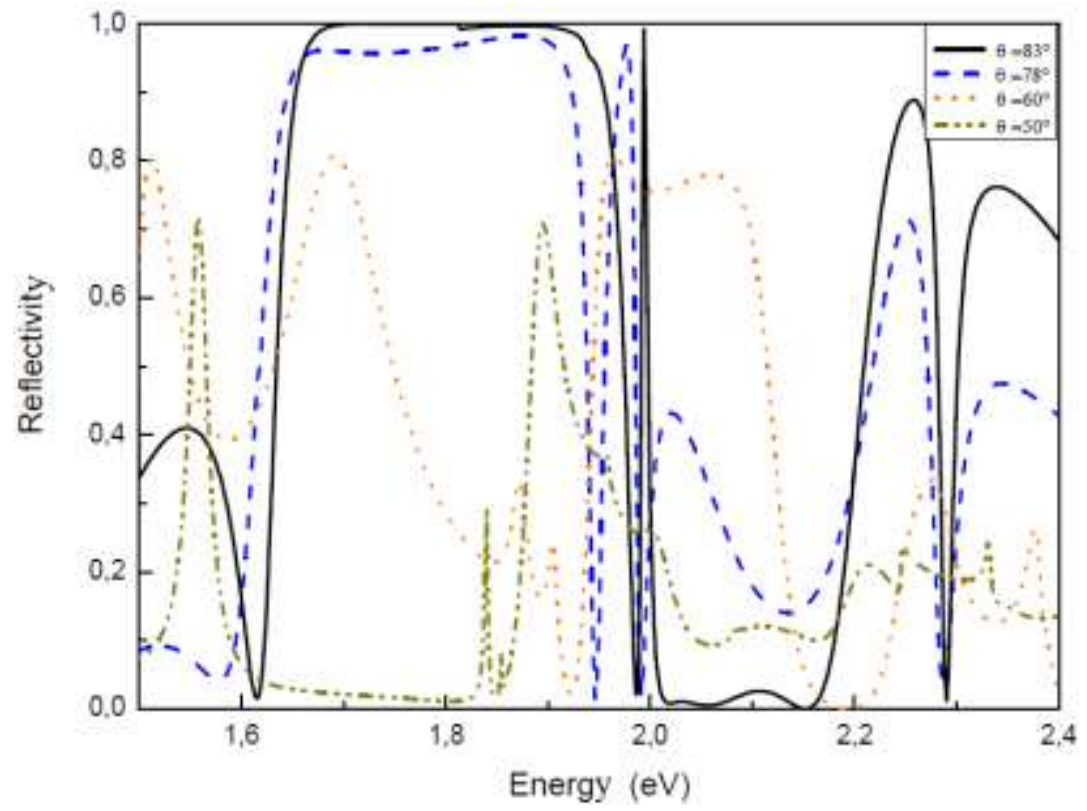

Fig.2:- Reflectivity spectra of semiconductor GaAs quantum grating for $L_{x}=\lambda / 4, h=350 \mathrm{~nm}, d=\lambda$ and $\varphi=\pi / 2$.

\section{Conclusion:-}

The knowledge of the optical properties of the photonic crystal is essential for applications in integrated optics. In particular, the photon mode control has been accomplished with localized mirrors such as Bragg reflectors. Nanostructured quantum wire grating constitute a waveguide with a high index contrast. To understand some properties of such structures, we conducted theoretical studies in order to evaluate various parameters characterizing the structure. Both at normal incidence and incidence parallel to the layers, this system behaves like nano-mirrors to a judicious choice of parameters of the structure and the angle of incidence $\theta$. It is considered as prototypes of onedimensional photonic crystals. In fact, the reflectivity spectra emphasize the opening of a photonic gap prohibiting the transmission of photonic modes on precise energy band. The opening of a mini-band transmission in the BIP signals the existence of allowed modes. This interesting property, comparable to those of photonic crystal defects, let these resonant systems are of major interest in the manufacture of lasers, waveguides and good quality filters.

\section{Acknowledgments:-}

We wish to express our sincere gratitude to Qassim University represented by the Deanship of Scientific Research for the materials and financial support provided in the course of this research. 


\section{References:-}

1. E. A. Muljarov, E. A. Zhukov, V. S. Dneprovskii and Yasuaki Masumoto, Phys. Rev. B 62, p 7420 (2000).

2. A. D'Andrea and R. Del Sole, Phys. Rev. B 48 (4), p2363 (1992).

3. L. Pilozzi, A. D’Andrea and H. Fenniche, Phys. Rev. B 64, p235319 (2001).

4. R. Charguia, H. Fenniche, R. Bennaceur, Physica E, Vol.40, pp. 770, (2008).

5. S. v Alfthan, F. Boxberg, K. Kaski, A. Kuronen, R. Tereshonkov, J. Tulkki and H. Sakaki, Phys. Rev. B 72, p 045329 (2005).

6. H. E. Ruda and A. Shik, Phys. Rev. B 72, p 115308 (2005).

7. M. P. Persson and H. Q. Xu, Phys. Rev. B 73, p 035328 (2006).

8. M. P. Persson and H. Q. Xu, Phys. Rev. B 73, p 125346 (2006). 